\title{
A Novel $\pi T N$ Gene Variant c.95136T>G (p.Cys31712Trp) and Associated Clinical Characteristics in a Family With Suspected Hereditary Myopathy With Early Respiratory Failure
}

Yoomi Yeo $\mathbb{C}^{0}$, M.D. $^{1 *}$, Jong Eun Park $\odot$, M.D., Ph.D. ${ }^{2 *}$, and Hyuk Sung Kwon $\oplus$, M.D., Ph.D. ${ }^{3}$

Departments of ${ }^{1}$ Internal Medicine, ${ }^{2}$ Laboratory Medicine, and ${ }^{3}$ Neurology, Hanyang University Guri Hospital, Hanyang University College of Medicine, Guri, Korea

Dear Editor,

Pathogenic variants of TTN have been reported to cause various myopathies, including hereditary myopathy with early respiratory failure (HMERF; OMIM 603689) [1]. As TTN is a large gene [2], its variants may cause diverse phenotypes. We report a family with a novel, likely pathogenic variant of TTN and mild clinical symptoms that manifested at old age.

This study was approved by the Institutional Review Board of Hanyang University Guri Hospital (2021-05-032) and informed consent was obtained from the patient. Experiments were conducted according to the Declaration of Helsinki. The proband (II-5), a 69-year-old male, was admitted to Hanyang University Guri Hospital, Guri, Korea, for vitrectomy in December 2019. He had hypertension and diabetes mellitus. He reported frequent arousal during sleep, without any respiratory symptoms. At admission, oxygen saturation was $85 \%$, arterial $\mathrm{pH}$ was 7.28 , arterial carbon dioxide partial pressure $\left(\mathrm{PaCO}_{2}\right)$ was $74.6 \mathrm{~mm}$ $\mathrm{Hg}$, and arterial oxygen partial pressure was $52.2 \mathrm{~mm} \mathrm{Hg}$. After the application of noninvasive ventilator support, arterial $\mathrm{PaCO}_{2}$ reached $48.3 \mathrm{~mm} \mathrm{Hg}$ and $\mathrm{pH}$ was 7.35.

Bilateral diaphragm elevation was revealed by chest computed tomography (Fig. 1A). A pulmonary function test showed a severe restrictive pattern (Fig. 1B and $1 \mathrm{C}$ ). Sniff test showed bilateral diaphragmatic paralysis (Fig. 1D and 1E). We observed a lack of movement on ultrasonography. Both echocardiography and electrocardiography monitoring were normal.

Six months later, the proband reported limb-girdle muscle weakness, but manual muscle strength was nearly normal (Medical Research Council grade 5). We found no hypertrophy or atrophy. Serum creatine kinase level was slightly elevated (3.98 $\mu k a t / L$ ). Nerve conduction was normal. Denervation potential was noted in thoracic paraspinal muscles and the right tibialis posterior on electromyography, but we detected no myopathic abnormalities.

The proband's 74-year-old sister (II-7) reported similar symptoms (Fig. 2A), including slowly progressive proximal and respiratory muscle weakness. Similar to the proband, her symptoms manifested at old age. She showed no definite muscle wasting or weakness. Additional information could not be obtained. The proband's mother (I-2) and brother (II-3) had similar clinical features, including respiratory failure, and they later died due to sudden cardiac arrest.
Received: August 18, 2020

Revision received: November 26, 2020

Accepted: May 6, 2021

Corresponding author: Hyuk Sung Kwon, M.D., Ph.D.

Department of Neurology, Hanyang University College of Medicine, Hanyang University Guri Hospital, 153 Gyeongchun-ro, Guri 11923, Korea

Tel: +82-31-560-2260, Fax: +82-31-560-2289

E-mail: kwonhs@hanyang.ac.kr

*These authors contributed equally to this study.

\section{(c) (1) (3)}

\section{(C) Korean Society for Laboratory Medicine}

This is an Open Access article distributed under the terms of the Creative Commons Attribution Non-Commercial License (https://creativecommons.org/licenses/by-nc/4.0) which permits unrestricted non-commercial use, distribution, and reproduction in any medium, provided the original work is properly cited. 
A

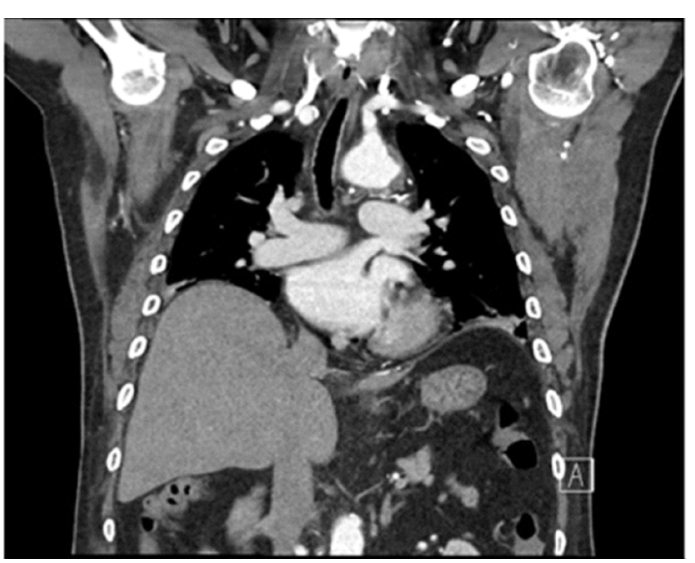

C

\begin{tabular}{|l|c|c|c|}
\hline & Predicted & Measured & $\% *$ \\
\hline FEV1, L & 2.59 & 0.82 & 32 \\
\hline FVC, L & 3.93 & 0.96 & 25 \\
\hline FEV1/FVC, \% & 73 & 86 & \\
\hline
\end{tabular}

(D)

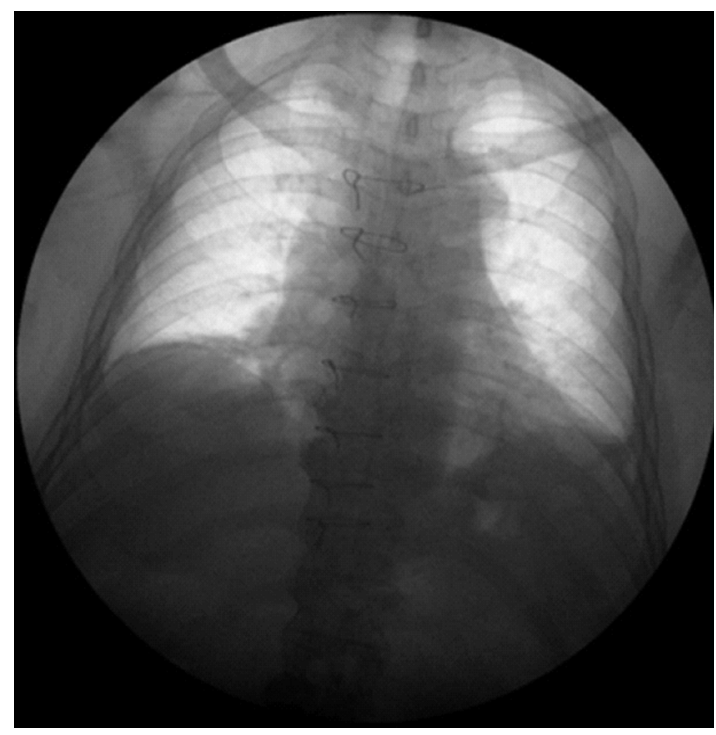

B

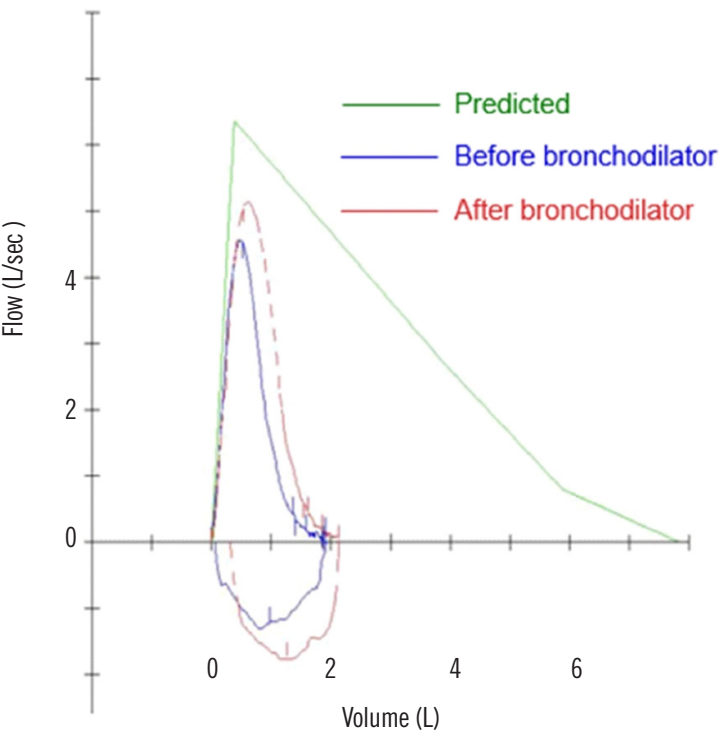

E

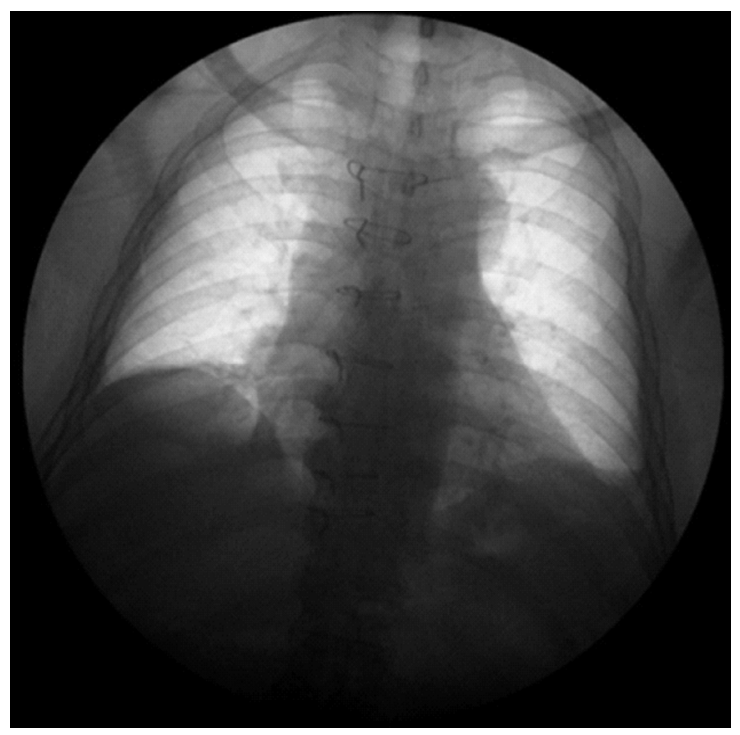

Fig. 1. Chest images and results of pulmonary and diaphragm function tests. (A) Chest computed tomography of the proband revealed bilateral diaphragm elevation. (B, C) Pulmonary function test showed a severe restrictive pattern, with negative bronchodilator test result. Chest X-ray was performed at full inspiration (D) and full expiration (E). Sniff test showed bilateral diaphragmatic paralysis. ${ }^{*}$ Percentage of measured value compared with predicted value.

Abbreviations: FEV1, forced expiratory volume in one second; FVC, forced vital capacity.

Genomic DNA was extracted from peripheral blood of the proband and the proband's sister.

Whole-exome sequencing for the proband's sister at the Hospital of the University of Pennsylvania, Philadephia, USA, in January 2020 revealed a novel heterozygous NM_001267550.1: c.95136T > G (p.Cys31712Trp) (or NP_001243779.1:p.Cys300 71Trp) TTN variant. Through Sanger sequencing, we found the same heterozygous TTN variant in the proband (Fig. 2B). The
p.Cys31712Trp variant was absent in large population databases, i.e., gnomAD (https://gnomad.broadinstitute.org/), the Korean Reference Genome Database (http://coda.nih.go.kr/coda/KRGDB/), ClinVar (https://www.ncbi.nlm.nih.gov/clinvar/), and the Human Gene Mutation Database Professional (version released in 2018; http://www.hgmd.org/), at the time of this study. The variant is located in the 119th fibronectin-3 domain (FN3 119) in the Aband region of TTN, where all HMERF-associated TTN patho- 
A

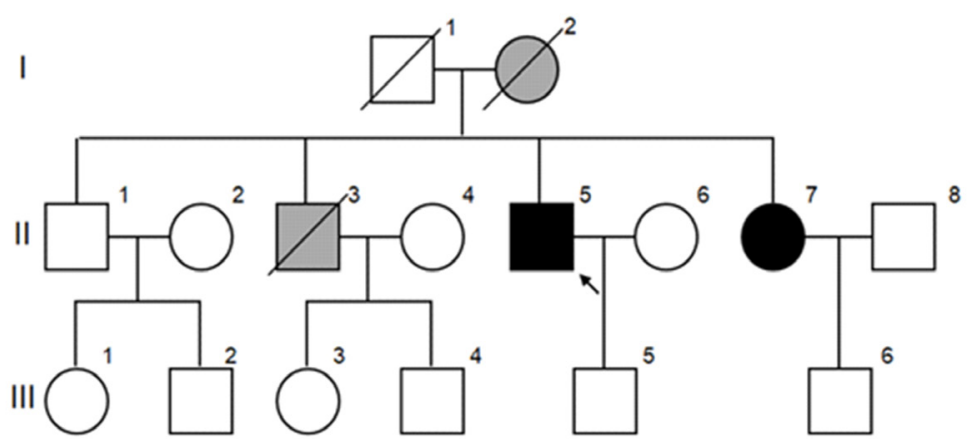

B

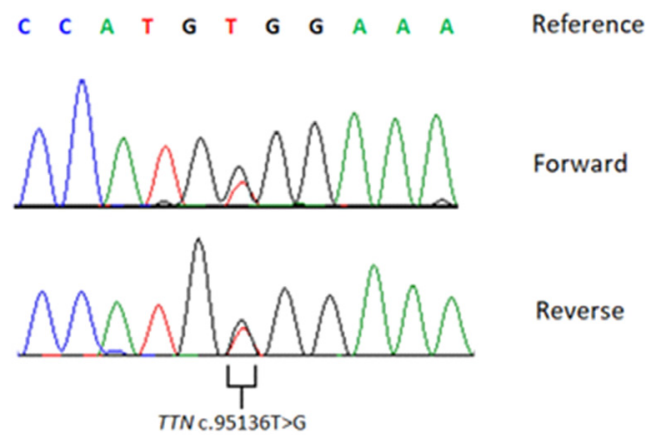

Fig. 2. Pedigree and sequence chromatogram of the novel TTN variant identified in the family. (A) Family pedigree. Open symbols indicate no signs or symptoms of hereditary myopathy with early respiratory failure (HMERF). Filled symbols represent affected individuals. Grey symbols indicate individuals who likely carry but were not tested for the TTN variant. The arrow indicates the proband. (B) Results of the likely pathogenic variant of TTN for the proband. Sequencing analysis revealed the NM_001267550.1:c.95136T>G (p.Cys31712Trp) (or NP_001243779.1:p.Cys30071Trp) variant.

genic variants are located [3]. The p.Cys31712Trp variant is located at the same amino acid position as p.Cys31712Arg (or NP_001243779.1:p.Cys30071Arg), the most common variant in HMERF [4]. This variant was also predicted to be deleterious in in-silico analysis using SIFT (https://sift.bii.a-star.edu.sg/) and Polyphen-2 (http://genetics.bwh.harvard.edu/pph2/). Based on our results, the TTN p.Cys31712Trp variant was considered a "likely pathogenic" variant, according to the 2015 American College of Medical Genetics and Genomics and the Association for Molecular Pathology guidelines [5, 6].

The relationships between individual pathogenic variants of TTN and different phenotypes have not been fully identified. Usually, disease onset due to pathogenic variants of TTN is from early to young adulthood and is characterized by respiratory failure or lower limb weakness [1, 7]. The clinical features of the patients in our study were compatible with HMERF [8]. The mean age of HMERF onset ranged from 16 to 53 years [7]. The degree and progression rate of muscle weakness are variable. However, to our knowledge, respiratory insufficiency at the age of 69 years without definite limb muscle atrophy or weakness has not been reported previously. Interestingly, the first symptom of HMERF in elderly patients may be respiratory failure caused by diaphragmatic paralysis.

The p.Cys31712Arg variant is the most common variant in HMERF and impairs proper folding of the FN3 119 domain [9]. The p.Cys31712Trp variant found in the current study may affect protein function and be related to a different phenotype. Titin is a giant structural muscle protein encoded by TTN $[1,8]$. It comprises four structurally and functionally distinct regions that interact with numerous protein partners [10]. This implies that different pathogenic variants can have diverse biological effects, which may cause various phenotypes, including those seen in heterogeneous muscular disorders.

We reported patients with a mild form of HMERF that may be associated with a novel, heterozygous, likely pathogenic variant of TTN. Evaluation of TTN may be helpful in patients with suspected hereditary muscular disorder-related respiratory failure. Further studies are needed to confirm whether this variant is pathognomonic and to reveal relationships between clinical manifestations and this TTN variant.

\section{ACKNOWLEDGEMENTS}

None declared.

\section{AUTHOR CONTRIBUTIONS}

All authors contributed to the conception and design of the study. Yeo $Y$ and Park JE contributed to data acquisition, analysis, interpretation, and drafting of the initial manuscript. Kwon HS contributed to data acquisition, interpretation, and revision of the manuscript for intellectual content. All authors have read and approved the final manuscript.

\section{CONFLICTS OF INTEREST}

The authors have no conflicts of interest to report. 


\section{RESEARCH FUNDING}

None declared.

\section{ORCID}

Yoomi Yeo

https://orcid.org/0000-0002-2447-2067

Jong Eun Park

https://orcid.org/0000-0001-9131-6518

Hyuk Sung Kwon

\section{REFERENCES}

1. Savarese M, Sarparanta J, Vihola A, Udd B, Hackman P. Increasing role of titin mutations in neuromuscular disorders. J Neuromuscul Dis 2016; 3:293-308.

2. Bang ML, Centner T, Fornoff F, Geach AJ, Gotthardt M, McNabb M, et al. The complete gene sequence of titin, expression of an unusual approximately $700-\mathrm{kDa}$ titin isoform, and its interaction with obscurin identify a novel Z-line to I-band linking system. Circ Res 2001;89:1065-72.

3. Pfeffer $G$ and Chinnery PF. Hereditary myopathy with early respiratory failure. In: Adam MP, Ardinger HH, et al., eds. GeneReviews ${ }^{\circledR}$ [Internet].

Seattle, WA: University of Washington, Seattle, 2014 [updated 2020 Mar 19].

4. Palmio J, Leonard-Louis S, Sacconi S, Savarese M, Penttilä S, Semmler $\mathrm{AL}$, et al. Expanding the importance of HMERF titinopathy: new mutations and clinical aspects. J Neurol 2019;266:680-90.

5. Richards S, Aziz N, Bale S, Bick D, Das S, Gastier-Foster J, et al. Standards and guidelines for the interpretation of sequence variants: a joint consensus recommendation of the American College of Medical Genetics and Genomics and the Association for Molecular Pathology. Genet Med 2015;17:405-24.

6. Kim YE, Ki CS, Jang MA. Challenges and considerations in sequence variant interpretation for Mendelian disorders. Ann Lab Med 2019;39: 421-9.

7. Palmio J, Evilä A, Chapon F, Tasca G, Xiang F, Brådvik B, et al. Hereditary myopathy with early respiratory failure: occurrence in various populations. J Neurol Neurosurg Psychiatry 2014;85:345-53.

8. Tasca G and Udd B. Hereditary myopathy with early respiratory failure (HMERF): Still rare, but common enough. Neuromuscul Disord 2018; 28:268-76.

9. Hedberg C, Toledo AG, Gustafsson CM, Larson G, Oldfors A, Macao B. Hereditary myopathy with early respiratory failure is associated with misfolding of the titin fibronectin III 119 subdomain. Neuromuscul Disord 2014;24:373-9.

10. Chauveau C, Rowell J, Ferreiro A. A rising titan: TTN review and mutation update. Hum Mutat 2014;35:1046-59. 